\title{
LE VICISSITUDINI DI CEM \\ IN EUROPA: UNO SFORTUNATO \\ PRINCIPE TURCO
}

\section{Alberto Boscolo}

La morte di Maometto II, avvenuta il 3 Maggio 1481, apriva un aspro conflitto tra i suoi figli, Bâyazìd e Cem, per la successione al trono. Nei suoi trenta anni di regno Maometto II, che aveva conquistato Costantinopoli nel 1453 a soli 24 anni d'età e che aveva esteso il dominio turco con l'occupazione della Serbia, di Atene e Corinto nel 1458, di Tebe nel 1460, di Trebisonda nel 1462 e di Scutari, tolta ai Veneziani nel 1479, aveva rafforzato lo stato e creato una minaccia per l'Europa cristiana e per il Mediterraneo, particolarmente per Venezia. L'occupazione di Trebisonda, avvenuta dopo un assedio durato un mese, sottrata ai Comneno, aveva messo in difficoltà i mercanti genovesi, che avevano nella città case e fondaci e che avevano già perso i loro privilegi a Galata e a Pera. Ma se da una parte Maometto II era considerato dai Turchi come «padre delle conquiste e delle guerre sante), dall'altra una politica di gravose imposte, dovuta anche alle campagne militari, lo avevano reso poco gradito ai sudditi; la Cristianità soprattutto dopo l'assedio di Otranto nel 1480, da lui voluto, l'aveva dipinto come un secondo Attila, come «un flagello di Dio» e sperava in una politica nuova da parte del suo successore, in una politica d'equilibrio, di stasi delle conquiste. Dei due figli, Cem, era più aperto a una revisione del sistema finanziario creato dal padre e alla Cristianità, che contava in una 
sua ascesa al trono, la quale avrebbe forse portato ad un sollievo, ad una possibilità di ripresa di fronte alle continue incursioni turche e ad un ritorno al controllo dei commerci provenienti dall'Oriente, perso soprattutto dai Genovesi più che dai Veneziani, che si erano piegati ben presto ad un accordo pur di non restare soffocati nell'Adriatico'.

La contesa, trasformata in guerra civile, fra i due fratelli, Bāyazīd e Cem, nati rispettivamente da Mukrime Hatun e da Cicek Hatun, prima e terza moglie di Maometto II, veniva determinata dalla successione al trono, non da impostazioni politiche e di governo. Cem, che era più giovane del fratello, riteneva di dover ereditare il sultanato, in quanto era nato quando il padre era sovrano, mentre Bāyazid, pur essendo nato quando Maometto II era ancora principe ereditario, anteponeva le sue pretese all'eredità regale come primogenito. Alla morte del padre Cem non si trovava a Istanbul; preposto all'importante governatorato di Karaman nel 1474, a quindici anni d'età, si trovava a Konya in compagnia della madre, che l'aveva allevato, e di una cerchia di dotti e di uomini d'arme, anche di origine cristiana. Egli stesso acquisiva una profonda cultura, conosceva l'arabo, il persiano, il greco e il latino; più tardi imparava l'italiano e il francese, e, incline alla poesia, oltrechè alle scienze, lasciava, alla pari di altri sovrani e principi turchi, quali Babūr Shāh, il conquistatore dell'India, e lo stesso Maometto II, una raccolta di pregevoli versi. L'assenza di Cem da Istanbul consentiva a Bāyazid di occupare il trono, anche perchè il messag-

${ }^{1}$ Un'eccellente, anche se antiquata, biografia di Cem è quella di L. THUASNE, Djem Sultan, Paris 1892; utilissima e pregevole anche quella più recente di Ertaylan, Sultan Cem, Istanbul 1951. Su Maometto II è fondamentale F. BABINGER, Maometto II e il suo tempo, Torino 1957. Su Cem a Venezia cfr. A. Gallotta, «ll trattato turco-veneto del 12 Gennaio 1482", in Studia turcologica memoriae Alexii Bombaci dicata, Napoli 1982, pp. 219 e segg. Sullo stesso Cem cfr., inoltre, il breve articolo di R. RAINERO, «La prigionia e la morte del SuItano Gem in Italia» in If veltro, 2-4, 1979, pp. 119-122. Sui commerci in Oriente cfr. E. AsHTOR, «Pagamento in contanti e baratto nel commercio italiano d'oltremare (secoli XIV-XVII)" in Storia di Italia, Annali 6, Torino 1983, pp. 382 e segg. 
gero, che doveva recare a Konya la notizia della morte di Maometto II, veniva ucciso nella strada di Amasya da partigiani dello stesso Bãyazīd. Cem sapeva così con molto ritardo quanto era accaduto e, pur essendo appoggiato a Istanbul, dove giungeva il 21 Maggio 1481, da molti seguaci, fra i quali Gedik Ahmed Pascià, già primo ministro di suo padre, doveva ricorrere alle armi contro il fratello, non avendo altra scelta ${ }^{2}$.

Al suo rientro a Konya iniziava cosi una guerra, un duro conflitto, nel quale aveva una posizione di primo piano lo stesso Pascià. Quest'ultimo, che nel 1480 aveva guidato la spedizione turca contro Otranto, era un militare, proveniente dai giannizzeri; secondo alcuni era un greco convertito, forse un Paleologo, secondo altri, un albanese o un serbo, che alle arti della guerra non associava una grande sensibilità politica. Aveva combattuto contro le genti degli Akkōyunlu, guidando l'ala destra dell'esercito nella battaglia di Otlukbeli, aveva attaccato le colonie genovesi in Crimea, assalito la Morea e comandato la flotta a Cefalonia, Zante e Santa Maura, ma non si era distinto nella politica interna come l'altro primo ministro di Maometto II, Mehmed Pascià, che era stato governatore di Karaman prima di Cem, del quale era amico. Gedik Ahmed Pascià doveva pagare con la morte l'appoggio dato al pretendente al trono; catturato e accusato di ribellione e di manovre filo-occidentali, ve-

${ }^{2}$ Sulle mogli di Maometto II e sulle cause del contrasto fra i due fratelli cfr. E.B. SAPOLYO, Osmanli Sultanlari tarihi, Istanbul 1961, pp. 104 e 125. Su Karaman, sulla cultura di Cem e sugli avvenimenti di poco successivi alla morte di Maometto II cfr. Mufassal osmanli tarihi, Istanbul 1958, vol. II, p. 616, S.K. KARAALIOGUU, Turk edebiyati tarihi, Istanbul 1973, p. 633, Y. ÖZTUNA, Turkiye tarihi, Istanbul 1964, vol. IV, p. 146, cfr., inoltre, A. GALloTA, G. BOVA, «Documenti dell'Archivio di Stato di Venezia, concernenti il principe ottomano Gen", in Studi magrebini, Napoli 1980, Vol. XII, pp. 185 e segg., oltrechè sugli avvenimenti, sulla politica veneziana e sui rapporti fra Băyazid e Venezia, che avrebbe dovuto adoperarsi per la cattura di Cem. Cfr., inoltre, J. LEFORT, Documents grecs dans les Archives de Topkapi. Contribution à l'histoire de Cem Sultan, Ankara 1981, utilissimi accanto alla altra documentazione per la ricostruzione degli avvenimenti. Sono grato alla collega Anna Masala per la traduzione dei testi turchi e al collega Ziya Umur per quella dei documenti ottomani da me consultati, in parte inediti. 
niva fatto giustiziare da Bāyazìd il 18 Dicembre 1482 a Edirne. Pur con l'appoggio di un militare tanto valido Cem subiva una sconfitta nel 1481 a Yenisehir e, costretto ad abbandonare il territorio di Karaman, si rifugiava in Egitto con la madre che era serba, la moglie, i figli, Óguzhān e Murad, e un seguito di trecento persone. Prima di giungere al Cairo il 26 Settembre 1481, si fermava a Damasco nel palazzo di Ablah; al Cairo, veniva ospitato con tutto il suo seguito dal sovrano mamelucco Qait Bey, uomo di fine cultura, che doveva legare il suo nome a molti monumenti della città e che intravedeva in Bãyazìd un pericolo per l'indipendenza del suo stato. Nel corso del suo soggiorno in Egitto Cem, unico principe ottomano pellegrino, fra il Febbraio e il Marzo 1482, si recava alla Mecca e a Medina; con l'aiuto di Qait Bey, riprendeva poi la guerra contro il fratello, che nel frattempo aveva rafforzato la sua posizione?

La lotta fra i due fratelli si chiudeva con la sconfitta di Cem, che nel Giugno 1482 dopo uno sfortunato scontro, avvenuto in Cilicia, stava per cadere prigioniero delle truppe di Bāyazid; pur di non cadere nelle mani del fratello. Cem, che aveva il titolo di «sehzade», cioè di principe, con alcuni suoi seguaci inviava a Rodi un emissario per ottenervi ospitalità e appoggi da parte dei Cavalieri di San Giovanni, che allora presidiavano l'isola, destinata anni dopo, nel 1522, con Solimano II, a diventare possesso dei Turchi. Cem pensava di raggiungere l'Ungheria, dove Mattia Corvino, timoroso della minaccia turca, era propenso a sostenerlo in cambio di un accordo di futura pace per i confini del suo regno; l'occupazione turca della Bulgaria nel 1398 e del regno di Tessalonica nel 1430 servivano d'esempio. Le trattative con l'emissario di Cem, condotte con il Cancelliere dell'Ordine, Pierre d'Aubusson, portavano ad un accordo, per il quale Cem sarebbe stato ospitato a Rodi, accompagnato in Ungheria, e in cambio si sarebbe impegnato, conquistando il trono, a firmare un trattato di pacifica coesistenza con la Cristianità, rappresentata dallo stesso Ordine; il trattato avrebbe dovuto prevedere, ac-

${ }^{3}$ Cfr. ancora Y. ÓzTUNA, op. cit, vol. IV, p. 117. Sulla figura di Qait Bey cfr. F. Gabriel., Gli Arabi, Firenze 1966, p. 185. 
canto alla fine delle imprese turche contro l'Europa, la ripresa dei rapporti commerciali d'un tempo, dei quali si sarebbero avvantaggiate soprattutto Genova e Venezia. I Genovesi, infatti, dopo la perdita dei loro privilegi a Pera, Galata e Trebisonda, avevano rafforzato la loro base di Chio e, costretti a pagare imposte sulle merci provenienti dai territori conquistati dai Turchi, differentemente dai Veneziani che agevolati nelle tasse, imbarcavano le merci a Scutari e avevano minori spese di trasporto, avevano diminuito la loro attività. Molti Genovesi anzi avevano preferito trasferire il loro campo d'azione nella penisola iberica, dove già avevano loro case commerciali in attivo. Cem, accettando le proposte di Pierre d'Aubusson, raggiungeva Rodi con alcuni del suo seguito il 30 Giugno $1482^{4}$.

Prima di lasciare la Cilicia riceveva da un turco del seguito di Bāyazìd, Nasuh, un invito a sottomettersi; per hui era stato previsto l'esilio e Nasuh gli consigliava per scritto di recarsi nella località che era stata prescelta, ma Cem rifiutava e indirizzava una lettera al fratello, confermando il suo proposito di non arrendersi e, poichè i suoi figli, che erano piccoli, dovevano essere consegnati allo zio e da lui educati, si opponeva. Bāyazid, intanto, attraverso un altro turco del suo seguito, Necmuddin, veniva informato della situazione delle mogli e dei figli di $\mathrm{Cem}$, riceveva particolari sui suoi seguaci e veniva a conoscenza delle trattative da lui condotte con Pierre d'Aubusson. Quest'ultimo, quando Cem giungeva a Rodi con tutto il suo seguito, un centinaio di persone, da prima rispettava l'accordo, concedeva al fuggiasco l'ospitalità promessa e un salvacondotto, che gli dava le prerogative di un Cristiano, poi mutava atteggiamento e lo teneva presso di sè come un prigioniero. Bāyazid, infatti, informato dell'accordo di Cem con l'Ordine dì San Giovanni, interveniva tentando una mossa politica al fine di ottenere l'invio del fratello in Turchia, raggiungendo però l'effetto contrario. I tre punti dell'accordo stabilivano una procura al Gran Maestro

${ }^{4}$ Cfr. R. RAINERO, op. cit., p. 120. Il raffronto fra le fonti turche, soprattutto la cronaca di Sa'd üd-Din, e quelle occidentali, favorisce la tesi di una promessa non mantenuta da parte di Pierre d'Aubusson con una conseguente prigionia di Cem a Rodi. 
dell'Ordine per trattare la pace tra Cem e Bâyazĩd nella maniera più opportuna e con ampia delega, una dichiarazione rilasciata dallo stesso Cem, nella quale affermava di aver chiesto personalmente allo stesso Ordine di essere mandato da Rodi in Francia e da lì in Ungheria, un trattato, composto di vari articoli, nel quale il medesimo Cem sottoscriveva, nel caso di acquisizione del trono paterno o di una parte del territorio turco, l'obbligo di una pacifica coesistenza con gli stati cristiani. Per il rispetto di tale accordo, steso in tre atti, da lui firmati e muniti del suo sigillo, due persone del suo seguito si obbligavano a restare a Rodi come ostaggi'.

Dall'accordo si intravedevano già d'allora la debole posizione di Cem, che contava anzitutto sull'appoggio dei Cavalieri per raggiungere una pace dignitosa con il fratello, e la fortunata posizione dell'Ordine, che poteva disporre dello stesso Cem per ottenere invece vantaggi da Bāyazìd senza attendere la progettata conquista del trono da parte del ribelle. Bãyazìd, molto abile, inviava così, poco dopo l'arrivo di Cem a Rodi, un suo emissario, Ilyas, per trattare il trasferimento del fratello in Turchia; le trattative, iniziate da Ilyas con Pierre d'Aubusson, venivano proseguite da Hersekzade Ahmet e poi da Hűsseyn; quest'ultimo, non riuscendo a portar via da Rodi Cem, avendo a disposizione 40.000 fiorini, datigli da Bāyazìd al fine di un accordo, otteneva dall'Ordine, tramite il versamento di un assegno annuo di 45.000 ducati, la promessa di neutralizzare Cem e versava ai Cavalieri, a titolo di contributo per il mantenimento del principe, la somma che portava con sè, equivalente ai ducati del primo anno. Cosi Pierre d'Aubusson mutava il suo atteggiamento e, comprendendo l'importanza che Cem aveva per lo stato turco, considerava lo stesso principe come un prigioniero da usare per ogni eventuale trattativa con gli Ottomani. Lattività a favore di Bāyazỉd veniva proseguita da un suo fedele, Iskender, Pascià, che

${ }^{5}$ Cfr. ancora R. RAINERO, op. cit., p. 121 e su Nasuh il documento 5690 , conservato nell'Archivio di Topkapi. Sulle lettere di Cem, di Necmúddin e sulle relazioni indirizzate a Bãyazĩd cfr. i docc. $11988,4615,5681,5487,5798$ dello stesso Archivio. 
era stato suo precettore, nominato poi governatore della Bosnia; quest'ultimo, che era stato già a Rodi prima dell'arrivo di Cem e che non era riuscito a convincere i Cavalieri dell'Ordine a non accoglierlo, completava l'accordo stretto da Hüsseyn. Allo stesso Iskender Bãyazīd aveva affidato il compito di uccidere uno dei figli di Cem, Oguuzhān, che veniva assassinato, in modo da creare uno sconvolgimento nel fratello e fra i suoi seguaci proficuo per la sua cattura. Cem si trovava, intanto, come prigioniero in un forte di Rodi e Bāyazìd, non fidandosi della promessa dei Cavalieri e comprendendo l'uso che avrebbero potuto farne, stabiliva di farlo uccidere. A conoscenza di tutti i particolari, relativi al forte, descrittigli in una lettera dal Vicerè di Sicilia, Gaspare de Spes, che intendeva rendersi amici i Turchi, inviava a Rodi un sicario, che avrebbe dovuto avvelenarlo; il sicario veniva però catturato e sottoposto a tortura. La situazione diventava così difficile per Bāyazīd nei confronti dell'Ordine e per un controllo della situazione egli mandava a Rodi Haci Ibrahim, che gli inviava una relazione, nella quale lo informava del trasferimento in Francia di Cem, progettato dall'Ordine ${ }^{6}$.

Mentre Bāyazīd, deluso ordinava un'incursione in Ungheria, comandata da Yakup Pascià, come avviso a Mattia Corvino per non appoggiare Cem, negli stati cristiani si notava l'importanza di averlo come prigionero e si delineava l'opportunità di sottrarlo all'Ordine. Da parte sua l'Ordine, dopo l'invio del sicario a Rodi, riteneva più sicuro il trasferimento di Cem e stabiliva di condurlo con il suo seguito in Francia. Con il pretesto di accompagnarli in Ungheria Cem e il suo seguito, scortati da alcuni Cavalieri, guidati da Guy de Blanchefort, venivano imbarcati in due navi il 31 Agosto 1482. Băyazĩd veniva informato del trasferimento da alcune spie turche, e, quando Cem, il 15 Otto-

' Sulla missione di Ilyas e su quelle di Hersekzade Ahmet e di Hüsseyn cfr. docc. $6679,5493,11984,5457$ dell'Archivio cit. Sul contributo di 40.000 fiorini cfr. Y. ÓztunA, op. cit., vol. IV, p. 119 e il doc. 3286 dello stesso Archivio. Sulla missione di Iskender Pascià cfr. i docc. 1460 e 5494 sempre dello stesso Archivio e sull'incarico affidato a Iskender di assassinare Óguzhân cfr. il doc. 11983 ancora dell'Archivio cit., dal quale si traggono anche le notixie relative al vicerè di Sicilia, al sicario e a Haci Ibrahim, docc. 5680, 1461, 5800. 
bre dello stesso anno sbarcava a Villafranca, nei pressi di Nizza, aveva notizie da un funzionario della dogana di Galata, un italiano, Andrea, che nel porto di Costantinopoli aveva avuto informazioni da marinai che erano stati in Francia. Cem era sempre sorvegliato da varie persone di fiducia di Bāyazìd e le notizie, raccolte da Hüsseyn, lo stesso che era stato a Rodi, venivano rimbalzate al medesimo Bāyazìd, che non aveva abbandonato il progetto di farlo assassinare. Il 5 Febbraio 1483, essendo scoppiata la peste nel territorio di Nizza, i Cavalieri decidevano di trasferire $\mathrm{Cem}$ e il suo seguito, che per la peste si era ridotto a quaranta persone, in una località non colpita dalla moria. Cem lasciava cosi Nizza, da lui ricordata in una poesia, e veniva condotto a Tenda, quindi a Cuneo e a Savigliano, accolto dappertutto con solenni festeggiamenti e ospitalità; si vedeva in lui il turco amico dei Cristiani, nel quale erano riposte le speranze di pace. Da Cuneo Cem veniva condotto in Savoia e vi restava per un lungo periodo, ben sorvegliato dai Cavalieri. Nel castello del barone Jacques de Sassenage, dove Cem risiedeva, si aveva un romanzo sentimentale, che aveva riflessi in Europa: quello del suo amore per la figlia dello stesso barone, Philippine Hélène, ben presto avvolto da un alone di leggende. E, mentre il Papato e la Francía tentavano attraverso le vie diplomatiche di sottrarlo all'Ordine e di impossessarsene, Bãyazìd a sua volta si rivolgeva a Venezia, affinchè lo catturasse e glielo consegnasse, ma non ci riusciva. Pareva, invece; che i Re Cattolici, Ferdinando e Isabella, fossero riusciti a convincere il Gran Maestro dei Cavalieri di Rodi e avessero ottenuto da lui il trasferimento di Cem in Spagna; a questo fine anzi i sovrani avevano concesso allo stesso Cem il 15 Dicembre 1483 a Vitoria un salvacondotto, affinchè si recasse nelle loro terre con il suo seguito, con i suoi averi e con una scorta di Cavalieri dell'Ordine, ma il principe, chiamato nei documenti spagnoli Giengemio o Zizimo, restava in Savoia.

Bāyazìd si limitava a seguire gli eventi, inviando spie in Francia ed affidando a Hüsseyin l'incarico di tenerlo informato di tutto con continue relazioni stese in base alle notizie fornite dagli stessi confidenti. Cem stava nel castello dei Sassenage con le persone sopravvissute del suo seguito come ospite dei Cavalieri, in 
realtà come prigioniero, anche se in una prigione dorata. Il re di Francia, Carlo VIII, che avrebbe voluto tenerlo presso di sè, si avvantaggiava della sua presenza in Savoia per i suoi rapporti con l'impero turco; il Pontefice Innocenzo VIII, salito al trono papale nel Febbraio 1484, insisteva presso i Cavalieri sin dai primi anni del suo Pontificato per averlo a Roma, mentre Bãyazīd tesseva una rete di informatori turchi nelle principali nazioni europee, in modo da avere un quadro della situazione anche nei riflessi di Cem, che frattanto godeva di un vitalizio di 65.000 ducati d'oro, concessogli dal sovrano mamelucco d'Egitto, Qait Bey, e che aveva respinto l'offerta di un'assegnazione annua di un milione di akçe fattagli dal fratello in cambio della rinuncia al trono? Nell'Aprile del 1489 Innocenzo VIII riusciva a farsi consegnare Cem dall'Ordine e Cem giungeva a Roma il 13 del mese successivo; alla sua custodia il Papa destinava tre Cardinali e al suo mantenimento $\mathbf{1 6 . 0 0 0}$ fiorini all'anno. Poco dopo il suo arrivo, poichè i Cavalieri avevano sempre ricevuto da Bãyazỉd il contributo annuo versato per la neutralizzazione di Cem, il Pontefice iniziava un negoziato con lo stesso Bāyazìd, affinchè gli desse una somma allo stesso fine. A Roma si recava un inviato turco, Mustafà Bey e in Turchia un messo del Papa, al quale Bāyazĩd inviava in dono alcuni cavalli e la reliquia della lancia di Longino; cosi Cem restava come un ostaggio prezioso e Bāyazid doveva sottostare alla situazione vantaggiosa per il Pontefice. Ancora una volta Cem, considerato come un ospite, era in realtà succube in una prigione dorata, oggetto politico e di speranze. E tale rimaneva quando la tiara veniva presa da Ales-

${ }^{7}$ Sull'incursione in Ungheria $c f r$. la relazione conservata nell'Archivio cit., doc. 4705. La navi salpavano il $1^{\circ}$ Settembre $1482 \mathrm{cfr}$. Y Öztuna, op. cit., vol. V, pp. 119 e segg., che ricorda anche i versi di Cem su Nizza. Cfr., inoltre, R. RAINERO, op. cit., p. 121. Sulla relazione di Andrea cfr, il doc. 11986 e su quelle di Husseyin i docc. 11982, 6601, dell'Archivio cit. Sul salvacondotto spagnolo cfr. A. DE LA TORRE, Documentos sobre las relaciones internacionales de los Reyes Católicos, Barcelona 1949, vol. I, doc.84, pp. 358 e segg. Cfr., infine, sui vitalizi Múnse'atu’s Selâtiyn, vol. 1, pp. 291 e seg. Sulla situazione in Europa, anche nei riflessi di Cem, efr. le notizie date da Ismail, emissario di Davut Pascià, e il rapporto sullo stesso Cem, che si trovano nei docc. 2970 e 6068 dell'Archivio cit. 
sandro VI della famiglia dei Borgia, I'11 Agosto 1492. Mentre si trovava presso il $\mathrm{Papa}, \mathrm{Cem}$ indirizzava alla madre, che risiedeva al Cairo, una lettera satura di amarezza e di nostalgia e faceva segnalare al fratello un gentiluomo suo amico, parente del Pontefice, che si recava a Gerusalemme. Carlo vIII e Ferdinando il Cattolico, frattanto, tentavano di sottrarre Cem al Papa per condurlo in Francia o in Spagna; per le ambizioni mediterranee del primo e per la tranquillità dei territori italiani del secondo il possesso di Cem era indispensabile, ma i tentativi erano inutili. La Francia cercava allora l'alleanza di altri stati per rapire Cem e Bāyazìd ne veniva informato da una lettera riservata scritta da un vescovo, Nicolò Simon, e a lui sottratta ${ }^{8}$.

In quello stesso periodo Bāyazīd aveva informazioni sulla situazione del ducato di Milano, governato con molta difficoltà da Ludovico il Moro, su quella della Spagna e del Portogallo, sopratutto sulle flotte e sugli eserciti delle due nazioni, su quella di Venezia; le notizie, ricche di particolari, gli giungevano da varie spie turche, che risiedavano in varie città europee e che fingevano di essere mercanti o uomini d'affari di passaggio, o gli pervenivano da Cristiani largamente ricompensati, fra i quali era un certo Leonardo, che stava a Roma. Bāyazīd, intanto, versava al Papato annualmente 40.000 fiorini d'oro, affinchè Cem fosse controllato e non agisse contro il suo Paese. Quando Carlo VIII scendeva in Italia e giungeva a Firenze, occupandola il 17 Novembre 1494, Iskender Pascià tentava di convincere Cem a fuggire, approfittando della situazione, a fare atto di omaggio al sultano suo fratello e a ritornare a Costantinopoli. Iskender faceva perveni-

${ }^{8}$ Sull'arrivo di Cem a Roma, sui tre Cardinali destinati alla sua custodia, e sull'assegnazione dei fiorini $c f r$. ancora l'Archivio cit., doc. 5454. Lo stesso Archivio conserva i documenti $(2783,3285,6192)$ relativi ai rapporti fra il Papa e Bãyazìd. Sulla lancia di Longino, conservata in un tabernacolo, opera del Pinturicchio, andato distrutto nel 1606, cfr. Y. ÖzTUNA, op. cit., pp. 146 e seg. Sulle lettere scritte da Cem $c f r$. i docc. 11987 e 11985 dell'Archivio cit. e sulla politica dei sovrani di Francia e di Spagna il doc. 5904 sempre dello stesso Archivio, che conserva anche la lettera del vescovo Nicolò Simon, doc. E 5456. La madre di Cem si era rivolta al doge di Venezia, perchè aiutasse Cem nel 1488, ma inultimente. Cfr. A. GALloTtA, G. Bova, Documenti cit., pp. 188 e seg. La politica venziana era molto ambigua. 
re a Cem, attraverso persone di fiducia, una lettera, nella quale gli esponeva l'alleanza di Ludovico il Moro con la Francia, suggerita dalla sua debole posizione in Italia, la discesa di Carlo VIII, l'occupazione della fortezza toscana di Fivizzano da parte dei Francesi, la fuga di Piero e Giuliano dei Medici da Firenze, la sottomissione, infine, di Firenze e di Siena e il progetto dello stesso Carlo VIII di occupare Napoli, passando per Roma. Iskender, che era preoccupato, in quanto Cem poteva cadere nelle mani del re di Francia, inviava una relazione a Bāyazīd sulla situazione italiana e sui riflessi possibili sia sullo stesso Cem, sia sulla Turchia. Carlo VIII vagheggiava, infatti, una crociatta contro i Turchi, successiva alla conquista del napoletano, e Bāyazid ne era informato anche da Hersekzade Ahmet Pascià, che consigliava buoni rapporti con Venezia, propensa a difendere le sue prerogative nell'Adriatico di fronte all'invadenza francese. Nel Gennaio 1495 Carlo VIII entrava a Napoli, passando per Roma, dove spadroneggiava; il re di Napoli Alfonso abdicava e il suo erede Ferrandino veniva deposto.

Cem, catturato a Roma da Carlo VIII, lo seguiva a Napoli nella sua spedizione, ma le sue speranze di libertà, il suo sogno di recuperare il trono, i suoi ideali erano delusi; il sovrano vedeva in lui una pedina da usare nelle sue mire orientali e Cem segretamente faceva appello al re d'Ungheria e al doge di Venezia, affinche lo rapissero. Bãyazid, informato di questo appello, affidava un delicato incarico a un tale Donaldo, uno degli Italiani, che risiedevano con le loro famiglie ancora in Turchia e che erano numerosi a Galata e a Pera, anche se costretti a una vita difficile; Donaldo doveva partire da Vallona, recarsi a Napoli, a Roma e a Firenze e riferirgli attraverso Mustafà Bey, lo stesso che era stato a Roma e che l'attendeva al rientro a Vallona, quale era la situazione italiana e che cosa poteva accadere a Cem. Donaldo riusciva nel suo compito $e$, poichè esisteva realmente il pericolo in Europa di aiutare Cem, Bâyazìd interveniva presso il doge di Venezia contro di lui e, non riuscendo ad avere l'aiuto della repubblica, ritornava - pare - nell'idea di farlo assassinare. Il 25 Febbraio, mentre stava a Napoli, Cem che aveva 35 anni, veniva avvelenato, forse da emissari del Papa. La sua morte 
veniva annunciata a Bāyazìd da un informatore con una lettera scritta in caratteri arabi, che gli veniva portata a Costantinopoli, e personalmente da un gentiluomo del seguito del principe, Sian Bey, che con un altro gentiluomo dello stesso seguito, Celâl Bey, aveva tenuto il rito funebre. La Corte ottomana prendeva il lutto e lo stesso Bāyazìd, secondo l'usanza, indossava un turbante nero, faceva distribuire denaro ai poveri, ordinava la temporanea chiusura di tutti i negozi e di tutti i mercati e la celebrazione di riti funebri in tutte le moschee del Paese. Tramontava in Europa l'idea di una coesistenza pacifica fra Cristiani e impero ottomano attraverso un sultano diverso da Bāyazìd. Prima che Bāyazìd morisse i Turchi occupavano Durazzo nel 1502 e dopo alterne campagne la Moldavia a la Valacchia nel 1504; con Solimano II l'espansione turca non trovava ostacoli e aumentava con l'occupazione di Belgrado nel 1521, di Rodi nel 1522, di Buda nel 1529, di Tabris (Persia) nel 1534, di Chio nel 1566; il controllo dei traffici dell'Oriente, passato quasi interamente ai Turchi, nuoceva a Venezia, mentre con la via dell'Atlantico, scoperta da Cristoforo Colombo, si aprivano nuovi mercati, che favorivano i Genovesi, insediatisi nella penisola iberica9.

Poco tempo dopo la morte del fratello, Bāyazìd otteneva da Federico di Napoli l'autorizzazione a trasferirne in Turchia la salma e si recavano nel napoletano alcuni turchi, che si occupavano del trasporto. Il corpo, tolto dal sepolcro, veniva avvolto in tela incerata e deposto in una cassa di piombo, adornata di metalli dorati e riposta in una cassa di legno. Sulla cassa di legno veniva inchiodato un drappo di velluto nero, sul quale figu-

${ }^{9}$ Cfr. sulle notizie inviate a Bãyazĩd, A. Boscolo, «Milano e la Spagna all'epoca di Ludovico il Morom in Milano nell 'età di Ludovico il Moro, Milano 1983, p. 98. Su Leonardo e su Iskender Pascià $c f r$. i doce. 6061, 6069 dell'Archivio cit. Su Carlo VIII e Cem cfr. l' «Epistolario di Pietro Martire d'Anghiera” in Documentos inéditos para la historia de España, Madrid 1953, t. IX, doc. 149, pp. 275 e segg. RAINERO, op. cit., p. 122; sulla richiesta di Cem al re d'Ungheria e a Venezia, su Donaldo e sui rapporti con Venezia cfr. i docc. 6071, 5497,11981 dello stesso Archivio. Sulla lettera, relativa alla morte di Cem, cfr. il doc. 6109 sempre dello stesso Archivio e sulla morte, l'Epistolario cit., doc. 161, p. 301. 
ravano due scudi dorati, e sul drappo veniva stesa una coltre di broccato bordata di velluto rosso. Il trasporto della cassa avveniva sino al luogo di imbarco su un carro ridipinto e rimesso a nuovo, che veniva scortato dai Turchi, guidati da Ali Bey, costretti a molti disagi per via delle strade impervie. La sesta sura del Corano sul Giudizio Universale, sulla resurrezione, sul popolo di Dio e sui falsi profeti, che Cem conosceva a fondo nei commenti, ritornava forse in mente ai Turchi della scorta con il concetto della vanità delle cose umane ${ }^{10}$.

La salma di Cem trovava temporanea sepoltura a Mudanya. Nel 1499 Bāyazīd, al quale erano stati consegnati dagli accompagnatori i libri, i cavalli, gli oggetti personali, la scimmia e il papagallo del fratello, stabiliva che il principe trovasse definitiva sepoltura a Bursa. Già aveva perdonato le persone che l'avevano accompagnato nell'esilio, riamettendole nell'amministrazione e nella vita dello stato; inoltre si era occupato del matrimonio della nipote, Gevher Melik, figlia di Cem. La madre, Çiçek Hatun, che si era battuta per la causa del figlio, era morta di peste al Cairo l'anno prima. A Bursa la salma di Cem, del quale a Roma nel Vaticano restava il ritratto del Pinturicchio, veniva sepolta nello splendido mausoleo, costruito nel 1479 per Sultan Mustafà, suo fratello, nel giardino della moschea Muradiye. Pochi anni dopo, nel 1522, moriva l'altro figlio di Cem, Murad, nel corso della conquista turca di Rodi, dieci anni prima si era spento Bāyazīd. L'emistichio, «se ti dicono che il sultanato è eterno/quella è una menzogna", scritto da Cem e rivolto al fratello, lasciava un amaro riflesso".

${ }^{10}$ Le notizie sono state tratte dai Registri della Fabbrica di Castel Nuovo, reg. III, conservati nell'Archivio di Stato di Napoli, in parte distrutto dai bombardamenti americani nel corso della seconda guerra mondiale. Sulla sura $c f r$. la traduzione del Corano, curata da A. BAUSANI, Firenze 1961, pp. 89, 105.

" $C f r$. Y ÖzTUNA, op. cit., vol. IV, p. 149, MǗNSE'A TU's Selâtiyn, vol. I, p. 294, TÁCU'T Terärih, vol. II, p. 40, E.H. AYVERDI, Osmanli Mi'märisinde Fatih Devri, 855, 886 (1451-1481), Istanbul 1973, vol. III, pp. 161 e segg., M. Trivulzio della Somaclia, I Papi, Firenze 1926, p. 392 e O. Asl.anapa, «Les relations culturelles et artistiques turco-italiennes» in Atti del Simposio di ricerche e di studi per uno sviluppo scientifico dei rapporti italo-turchi, Milano 1981 , p. 167. 